\title{
A case of malignant melanoma after repeated recurrent dysplastic nevi
}

\author{
Dae Kyun Jeong ${ }^{1}$, \\ Yong Chan Bae ${ }^{1,2}$, \\ So Jeong Lee ${ }^{3}$, \\ Hoon Soo Kim ${ }^{4}$, \\ Young Jin $\mathrm{Choi}^{5}$ \\ ${ }^{1}$ Department of Plastic and \\ Reconstructive Surgery, Pusan National \\ University School of Medicine, Busan; \\ ${ }^{2}$ Biomedical Research Institute, Pusan \\ National University Hospital, Busan; \\ Departments of ${ }^{3}$ Pathology, \\ ${ }^{4}$ Dermatology, and ${ }^{5}$ Internal Medicine, \\ Pusan National University School of \\ Medicine, Busan, Korea
}

\begin{abstract}
Dysplastic nevus is common and affects about $10 \%$ of the northern European-descendent population. Studies over the past several decades have identified dysplastic nevi as a risk factor for malignant melanoma. Furthermore, in rare cases, they confirmed that dysplastic nevi have progressed to melanoma. Cases in which dysplastic nevi progressed to malignant melanoma in multiple studies are not uncommon. A 35-year-old woman presented with the major symptom of multiple itchy brown nodules $(2.0 \mathrm{~cm} \times 1.3 \mathrm{~cm})$ in the left cheek that had first appeared 20 years earlier. Complete excision was performed at the first visit; subsequent biopsy confirmed that they were dysplastic nevi. They recurred three times over 3 years at the same site, all of which were histologically diagnosed as dysplastic nevi. Five years after the final excision, a brownish nodule developed in the left cheek, with others at the left temporal region, right retroauricular region, and left shoulder at the same time. These lesions were histologically diagnosed as malignant melanoma. We experienced a case of malignant melanoma that occurred at the same site after three recurrences of dysplastic nevi. Although rare, the possibility of malignant melanoma should be considered in follow-ups in cases involving repeatedly recurrent dysplastic nevi.
\end{abstract}

Keywords: Dysplastic nevus syndrome / Melanoma / Recurrence

\section{INTRODUCTION}

Dysplastic nevus (DN) is relatively common and known to affect about $10 \%$ of the northern European-descendent population [1]. Studies over the past several decades have identified $\mathrm{DN}$ as a risk factor for malignant melanoma [2,3]. Furthermore, in rare cases, DN progresses to melanoma. In fact, cases in which DN progressed to malignant melanoma have been described in multiple studies [4]. However, cases in which DN repeatedly recur and a malignant melanoma develops in the same site are rarely found worldwide. Here we report a rare case in which malignant melanoma developed at the site where DN

\section{Correspondence: Yong Chan Bae}

Department of Plastic and Reconstructive Surgery, Pusan National University

School of Medicine, 179 Gudeok-ro, Seo-gu, Busan 49241, Korea

E-mail: baeyc2@hanmail.net

Received May 26, 2019 / Revised August 2, 2019 / Accepted August 11, 2019 recurred three times and was completely excised since the initial onset of DN that was also completely excised.

\section{CASE REPORT}

This study was approved by the Ethics Committee of Pusan National University Hospital prior to its start. Informed consent was obtained for the use of patient data. A 35-year-old woman presented to the hospital on March 9, 2007, with multiple itchy brownish nodules $(2.0 \mathrm{~cm} \times 1.3 \mathrm{~cm})$ on her left cheek that had first appeared 20 years earlier (Fig. 1). The patient had no family history of malignant melanoma. The nodules were diagnosed as compound nevi, which are benign tumors, in a previous biopsy. Therefore, in order to resect the nodules, we chose a maximum surgical margin of $1 \mathrm{~mm}$ close to the $\mathrm{DN}$. The lesion was completely resected and sutured, and a biopsy confirmed the 


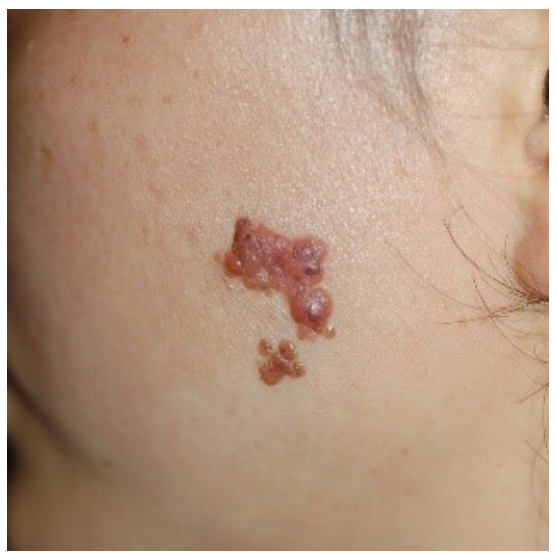

Fig. 1. Multiple itchy brownish nodules. Multiple itchy brownish nodules $(2.0 \mathrm{~cm} \times 1.3 \mathrm{~cm})$ in the left cheek that had first appeared 20 years prior.

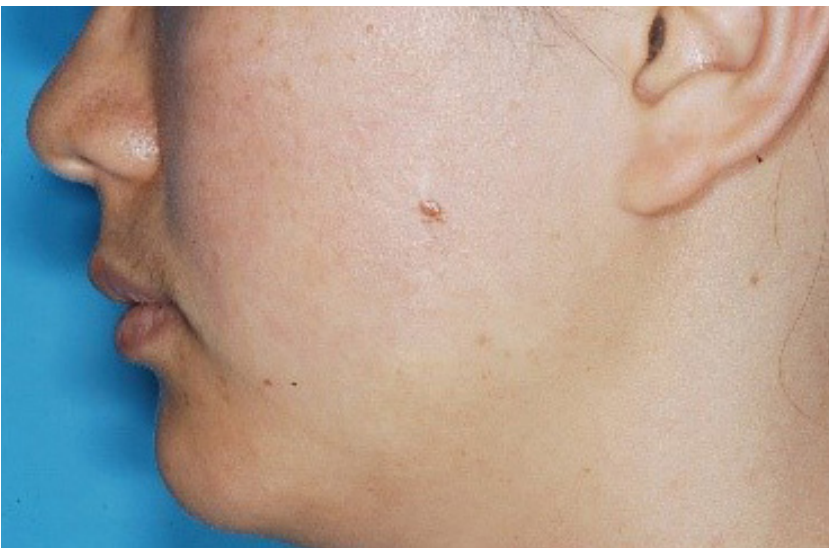

Fig. 2. First recurrence. A $0.5 \mathrm{~cm} \times 0.5 \mathrm{~cm}$ nodule that developed 15 months after the first excision.

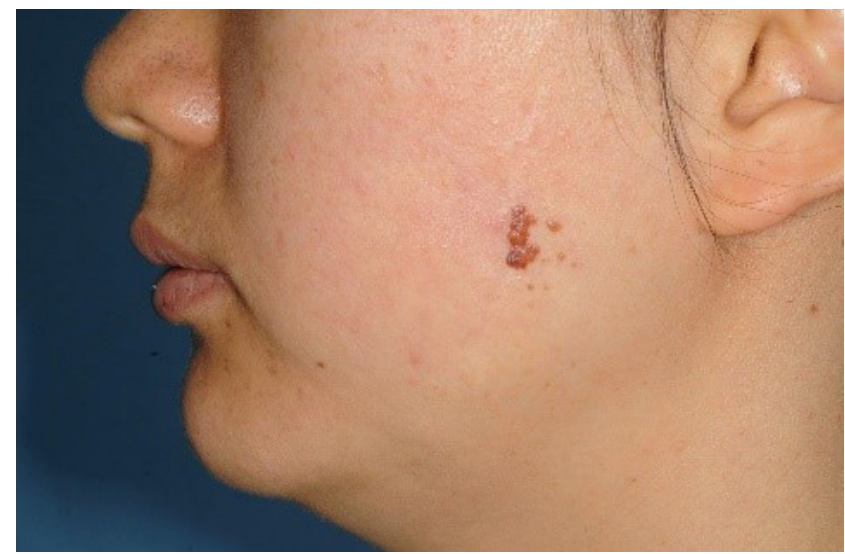

Fig. 3. Second recurrence. A $1.0 \mathrm{~cm} \times 0.5 \mathrm{~cm}$ nodule that developed 10 months after the second excision.

diagnosis of DN. Furthermore, the nodules were diagnosed as moderate atypia in accordance with the grading criteria for DN [5]. Fifteen months later on June 9, 2008, the patient visited the

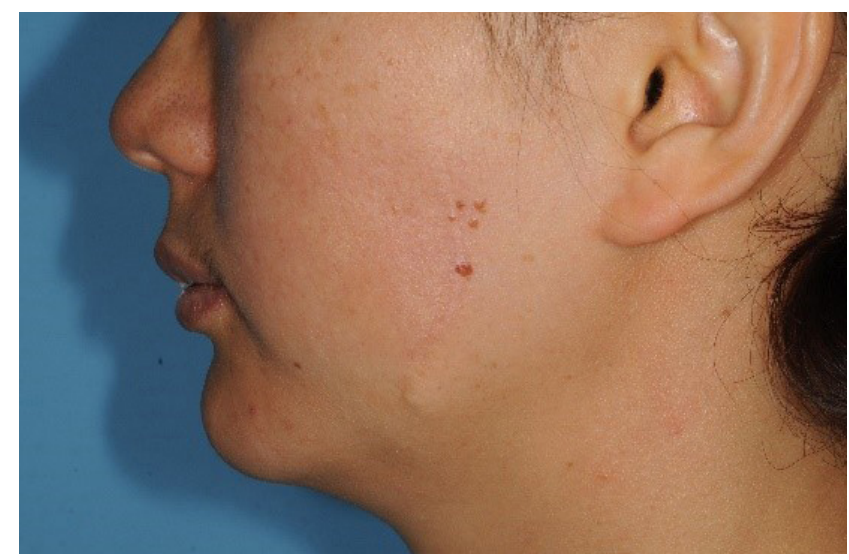

Fig. 4. Third recurrence. A $0.5 \mathrm{~cm}$ nodule that developed 9 months after the third excision.

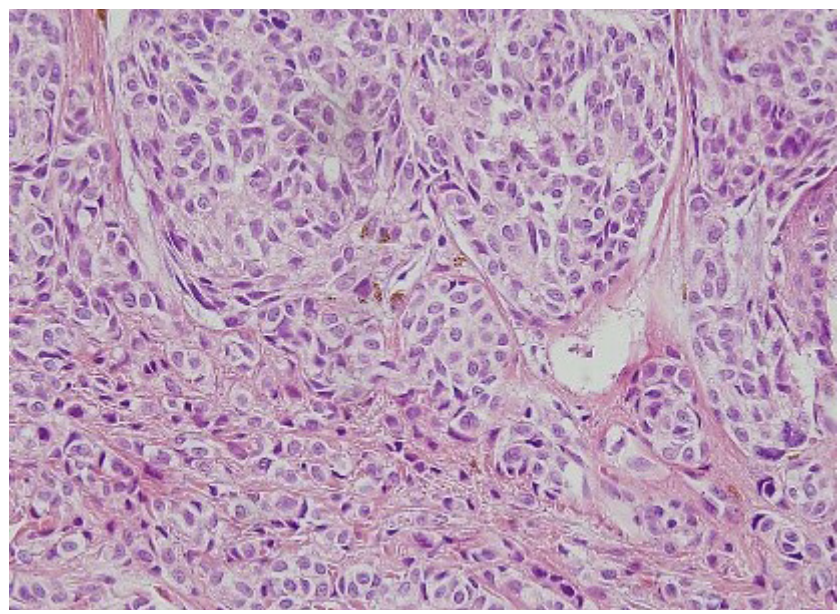

Fig. 5. Histopathologic findings of recurrent dysplastic nevus (third recurrence). Melanocytes: round, regular cells with moderate amounts of cytoplasm. No nuclear atypia, pleiomorphism, or mitosis are visible $(\mathrm{H} \& \mathrm{E}, \times 400)$.

hospital with a $0.5 \mathrm{~cm} \times 0.5 \mathrm{~cm}$ nodule at the same site (Fig. 2), which was also excised with a maximum surgical margin of 1 -mm close to DN. The lesion was again histologically diagnosed as DN with moderate atypia. Ten months later on April 13,2009 , the patient underwent excision of a $1 \mathrm{~cm} \times 0.5 \mathrm{~cm}$ nodule at the same site with a maximum surgical margin of 1-mm close to DN (Fig. 3) and again was histologically confirmed as DN with moderate atypia. Nine months later on January 8,2010 , the patient developed multiple brownish nodules at the same site and $0.5-\mathrm{cm}$ nodules on her left shoulder, which were subsequently excised with a maximum surgical margin of 1-mm close to DN (Fig. 4). The biopsy results suggested that some melanocytes formed a nest that was confined to the dermal-epidermal junction. The melanocytes showed no nuclear atypia, pleiomorphism, or mitosis. Immunohistochemical 

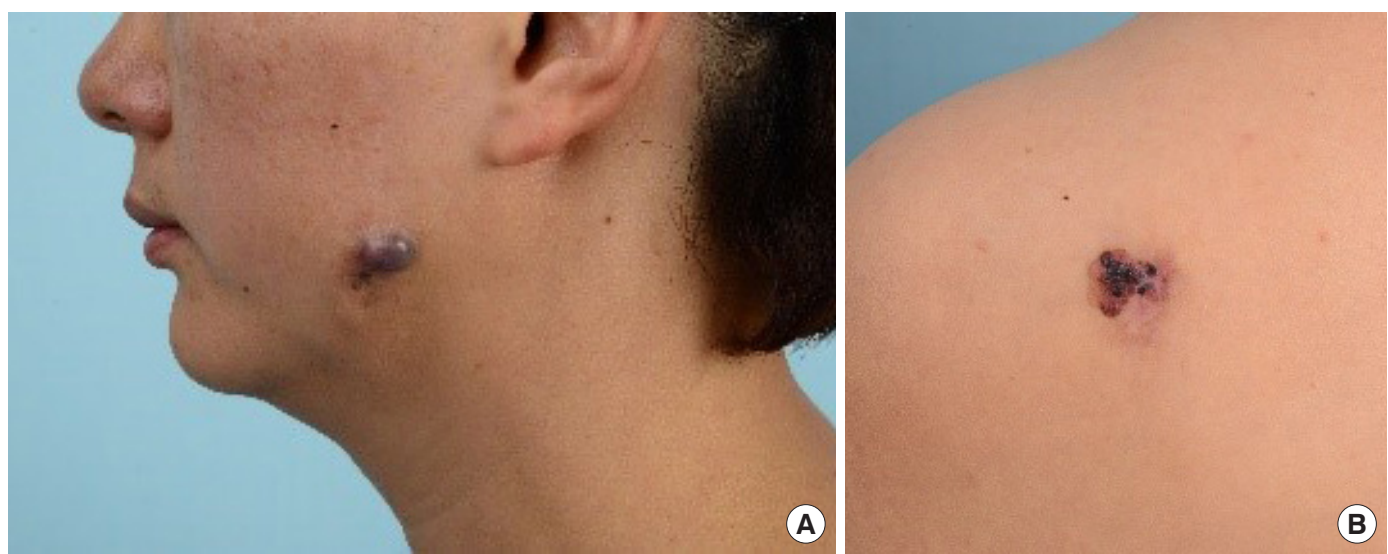

Fig. 6. Mass after three times of recurrence of dysplastic nevi. Mass in the left shoulder, left cheek, and right retroauricular region that developed 5 years and 9 months after the fourth excision, respectively. (A) Left cheek and (B) left shoulder.

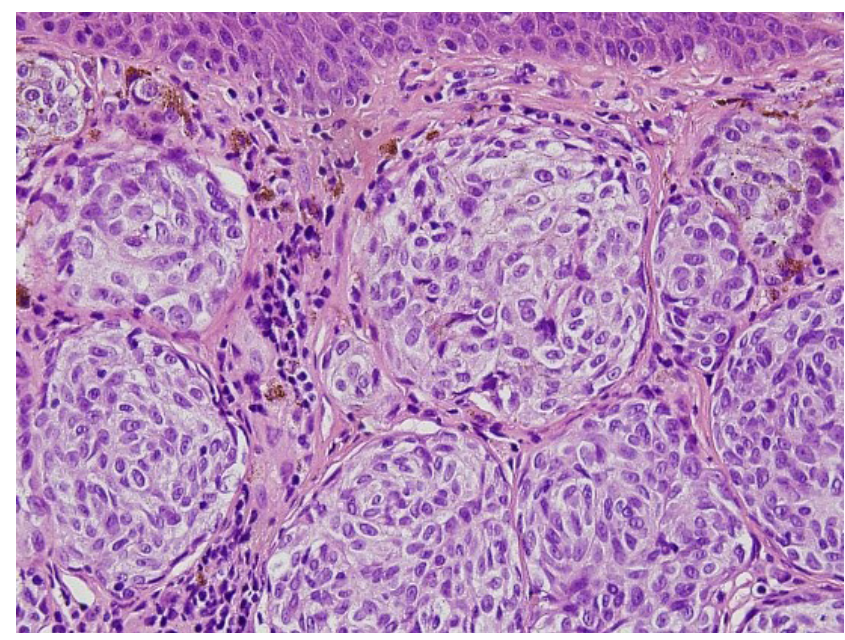

Fig. 7. Histopathologic findings of malignant melanoma after repeated recurrent dysplastic nevi. Tumor cell: round to oval nuclei showing mild nuclear pleomorphism with small nucleoli and irregular nuclear membrane. The cytoplasm contains melanin pigmentation. No mitosis is evident $(\mathrm{H} \& \mathrm{E}, \times 400)$.

staining confirmed S-100 and HMB-45 positivity, while immunohistochemical staining for HMB-45 showed strong staining of tumor cells in the superficial dermis but not in the deeper dermis. On the basis of these results, the lesions were diagnosed as DN with moderate atypia (Fig. 5).

At 5 years and 9 months after the final surgery, on October 27, 2015, the patient developed a mass in the left cheek, right retroauricular region, and left shoulder. Histopathological findings at another hospital confirmed that the lesions in the left cheek and right retroauricular region were malignant melanoma and lesion in the left shoulder was compound nevi. On the preoperative positron emission tomography-computed tomography (PET-CT), glucose uptakes are only found in the right retroauricular region and left cheek. No glucose uptake was observed in other areas. On the preoperative abdominal CT, no evidence of metastasis was found.

The patient underwent tumor excision in the left temporal and right retroauricular regions, left cheek, and left shoulder at our hospital (Fig. 6). For the left temporal region, left cheek, right retroauricular region, and left shoulder, frozen biopsy was performed to determine the surgical margin. After confirmation of the tumor-free margin (left temporal region, $0.5 \mathrm{~cm}$; left cheek, $0.5 \mathrm{~cm}$; right retroauricular region, $1 \mathrm{~cm}$; left shoulder, 2 $\mathrm{cm}$ ), split-thickness skin graft was performed for the left temporal region, right retroauricular region, and left shoulder, and a local advancement flap was applied on the left cheek. The histopathological findings confirmed features suggestive of malignant cells, such as prominent nucleoli, vesicular chromatic pattern, increased nucleus-to-cytoplasm ratio, irregular nuclear membrane, and nuclear pleomorphisms, in the left temporal region, right retroauricular region, left cheek, and left shoulder. Furthermore, immunohistochemical staining showed MelanA and HMB-45 positivity, with elevated Ki-67 proliferation, based on which the patient was diagnosed with stage IV (T4a N0 M1a) melanoma (superficial spreading type) (Fig. 7). The Breslow thickness measurements were as follows: left cheek, $6 \mathrm{~mm}$; left temporal region, $6 \mathrm{~mm}$; right retroauricular region, $8 \mathrm{~mm}$; left shoulder, $2 \mathrm{~mm}$ ).

Two months later, on December 18, 2015, nevi developed in the forehead, abdomen, and left shoulder and were subsequently excised. The patient was diagnosed with melanoma in situ at the forehead, intradermal melanocytic nevus at the left shoulder, and hyperpigmentation without neoplastic change at the abdomen.

The patient underwent chemotherapy with high-dose interferon from December 2015 to December 2016. Since then, the patient has not shown recurrence and is currently being fol- 
lowed up at our plastic surgery and hemato-oncology departments.

\section{DISCUSSION}

There is much debate regarding the definition of DN. The Dutch Working Group [6] used the following clinical criteria for $\mathrm{DN}$ : $\geq 5 \mathrm{~mm}$ in diameter, vague border, asymmetric shape, irregular pigmentation, and red hue.

Evidence is mounting that the risk for melanoma is increased in patients with DN. Multiple studies have shown that DN is an independent risk factor for multiple primary melanoma $[2,3]$. Gandini et al. [7] reported in a meta-analysis that the incidence of melanoma increases tenfold in the presence of $\mathrm{DN}$.

Although patients with DN are known to be at an elevated risk of melanoma, it is difficult to argue that the incidence of melanoma is higher with DN than with common nevi. In their study on melanoma kindreds, Tucker et al. [8] confirmed that most DN cases were regressed or stabilized and only a few cases progressed to melanoma. Furthermore, several studies found that DN can act as a precursor to sporadic melanoma [4].

$\mathrm{DN}$ can act as a precursor to melanoma, but it is difficult to conclude that it more commonly progresses to melanoma than does common nevi. In a study of 147 cases of melanoma that progressed from nevi, Skender-Kalnenas et al. [9] found that $56 \%$ of them originated from $\mathrm{DN}$ while $44 \%$ originated from common nevi. Bevona et al. [10] studied 1,606 cases of nevusassociated melanoma and found that $43 \%$ originated from DN while $57 \%$ originated from common nevi. In a study spanning 10 years, Goodson et al. [11] reported that 66 cases of melanoma originated from common nevi, while 45 cases progressed from DN. In other words, many studies found comparable rates of DN and common nevi progressing to melanoma.

Once $\mathrm{DN}$ is confirmed, total body photography is crucial to confirm changes to the nevi. Although DN changes over time [8], most cases achieve involution. Excision is not recommended for all cases of DN, and excisional biopsy should be performed only when melanoma is suspected.

Prophylactic removal of all nevi is inappropriate since very few nevi progress to melanoma and these changes are unpredictable [1]. Further, the risk of melanoma persists even with removal of all nevi due to the possible development of de novo melanoma.

One of the most controversial areas remains whether to observe or re-excise moderately dysplastic nevi that were excisionally biopsied without clinical residual pigment but with positive histological margins. In 2015, the Pigmented Lesion Subcommittee published a consensus statement with the following con- clusions: (1) mildly and moderately dysplastic nevi with negative margins do not warrant re-excision; (2) mildly dysplastic nevi biopsied without clinical residual pigment but with positive histological margins may be safely monitored rather than re-excised; and (3) observation may be a reasonable option for the management of moderately dysplastic nevi with positive histological margins [12].

In this case, $\mathrm{DN}$ with moderate dysplasia recurred three times. In accordance with the guideline, close observation is a reasonable choice. However, considering the ambiguity of DN, treatment might be considered in accordance with the malignant melanoma.

In our case, $\mathrm{DN}$ recurred three times in the same site after complete excision of the initial DN in the left cheek; all nodules were completely excised. However, when it recurred for the fourth time, the lesion was confirmed to be malignant melano$\mathrm{ma}$, and the DN seems to have acted as a precursor.

Recurrent nevus is defined as the appearance of a melanocytic lesion in a site from which a previous benign nevus was removed [13]. It generally affects women in their 20s and 30s and most commonly occurs in the back. Cases involving the facial area are relatively rare [14]. Recurrence commonly occurs within 6 months of excision [14]. Although there is no consensus on the type of nevus that most commonly recurs, a study of recurrent nevi found that $20 \%-30 \%$ of cases were DN [14]. In contrast to recurrent nevi, Blum et al. [15] found that recurrent malignant melanoma commonly occurs in people in their 30s or older and that recurrence takes longer time. Unlike general cases of recurrent nevi, our patient had recurrence in the facial area, and the time until recurrence was relatively long at 15 months, 10 months, and 9 months, respectively.

Here we described a case of malignant melanoma that occurred at the same site after three recurrences of DN. Although rare, the possibility of malignant melanoma should be considered in the follow-up of cases involving repeatedly recurrent dysplastic nevi.

\section{NOTES}

\section{Conflict of interest}

No potential conflict of interest relevant to this article was reported.

\section{Ethical approval}

The study was approved by the Institutional Review Board of Pusan National University Hospital (IRB No. H-1905-017-079) and performed in accordance with the principles of the Declaration of Helsinki. Written informed consent was obtained. 


\section{Patient consent}

The patient provided written informed consent for the publication and the use of her images.

\section{ORCID}

Dae Kyun Jeong https://orcid.org/0000-0002-6379-2629

Yong Chan Bae https://orcid.org/0000-0002-0268-4667

So Jeong Lee https://orcid.org/0000-0002-6465-9811

Hoon Soo Kim https://orcid.org/0000-0002-7649-1446

Young Jin Choi https://orcid.org/0000-0003-1030-4211

\section{REFERENCES}

1. Tucker MA. Melanoma epidemiology. Hematol Oncol Clin North Am 2009;23:383-95.

2. Carey WP Jr, Thompson CJ, Synnestvedt M, Guerry D 4th, Halpern A, Schultz D, et al. Dysplastic nevi as a melanoma risk factor in patients with familial melanoma. Cancer 1994;74: 3118-25.

3. Burden AD, Newell J, Andrew N, Kavanagh G, Connor JM, MacKie RM. Genetic and environmental influences in the development of multiple primary melanoma. Arch Dermatol 1999;135:261-5.

4. Masri GD, Clark WH Jr, Guerry D 4th, Halpern A, Thompson CJ, Elder DE. Screening and surveillance of patients at high risk for malignant melanoma result in detection of earlier disease. J Am Acad Dermatol 1990;22(6 Pt 1):1042-8.

5. Weinstock MA, Barnhill RL, Rhodes AR, Brodsky GL. Reliability of the histopathologic diagnosis of melanocytic dysplasia. The Dysplastic Nevus Panel Arch Dermatol 1997;133:953-8.

6. Bergman W, van Voorst Vader PC, Ruiter DJ. Dysplastic nevi and the risk of melanoma: a guideline for patient care. Nederlandse Melanoom Werkgroep van de Vereniging voor Integrale Kankercentra. Ned Tijdschr Geneeskd 1997;141:2010-4.
7. Gandini S, Sera F, Cattaruzza MS, Pasquini P, Abeni D, Boyle P, et al. Meta-analysis of risk factors for cutaneous melanoma: I. common and atypical naevi. Eur J Cancer 2005;41:28-44.

8. Tucker MA, Fraser MC, Goldstein AM, Struewing JP, King MA, Crawford JT, et al. A natural history of melanomas and dysplastic nevi: an atlas of lesions in melanoma-prone families. Cancer 2002;94:3192-209.

9. Skender-Kalnenas TM, English DR, Heenan PJ. Benign melanocytic lesions: risk markers or precursors of cutaneous melanoma? J Am Acad Dermatol 1995;33:1000-7.

10. Bevona C, Goggins W, Quinn T, Fullerton J, Tsao H. Cutaneous melanomas associated with nevi. Arch Dermatol 2003;139: 1620-4.

11. Goodson AG, Florell SR, Boucher KM, Grossman D. A decade of melanomas: identification of factors associated with delayed detection in an academic group practice. Dermatol Surg 2011; 37:1620-30.

12. Kim CC, Swetter SM, Curiel-Lewandrowski C, Grichnik JM, Grossman D, Halpern AC, et al. Addressing the knowledge gap in clinical recommendations for management and complete excision of clinically atypical nevi/dysplastic nevi: Pigmented Lesion Subcommittee consensus statement. JAMA Dermatol 2015;151:212-8.

13. Schoenfeld RJ, Pinkus H. The recurrence of nevi after incomplete removal. AMA Arch Derm 1958;78:30-5.

14. King R, Hayzen BA, Page RN, Googe PB, Zeagler D, Mihm MC Jr. Recurrent nevus phenomenon: a clinicopathologic study of 357 cases and histologic comparison with melanoma with regression. Mod Pathol 2009;22:611-7.

15. Blum A, Hofmann-Wellenhof R, Marghoob AA, Argenziano G, Cabo H, Carrera C, et al. Recurrent melanocytic nevi and melanmas in dermoscopy: results of a multicenter study of the International Dermoscopy Society. JAMA Dermatol 2014;150: 138-45. 